\author{
ARIADNA STRUGIELSKA \\ Department of English, Nicolaus Copernicus University, Torun, Poland \\ ariadnas@umk.pl
}

\title{
BETWEEN GALILEO AND DARWIN, OR TOWARDS A UNIFIED MODE OF IDEALIZATION IN COGNITIVE LINGUISTICS
}

\begin{abstract}
The aim of this paper is to demonstrate that Cognitive Linguistics is in need of a coherent model through which the meaning of symbolic units can be represented. Departing from the premise that scientific theorizing requires taking a stance on the nature of idealization, the current discussion concentrates on cognitive linguists' perspectives on the process, and reveals a lack of uniformity in the models proposed.

On the whole, generalizations in Cognitive Linguistics are conducted in a manner reflecting the basic commitments of the approach, and hence the idealized models discussed strive to capture the embodied nature of cognition reflected in the semantic poles of symbolic units. However, a detailed analysis shows that a number of significant choices underlying the process of abstraction are random. Consequently, the modes of idealization revealed through semantic frames and cognitive domains lead to the emergence of barely compatible semantic categories which are assumed to represent the meaning of the same symbolic unit.

It is thus postulated that if Cognitive Linguistics aspires to become a mature scientific theory, whose constructs and hypotheses can be operationalized and falsified, it needs to develop a more unified framework in which the situated and distributed natures of meaning are accounted for in a motivated manner.
\end{abstract}

Keywords: cognitive linguistics; semantic theorizing; situated and distributed meaning; idealization

\section{Introduction}

In sciences where top-down generalizations are tested with bottom-up evidence, and in disciplines, such as linguistics, which seek to find a balance between deductive principles and inductive observables, theories are built through the process of idealization. This process involves presenting phenomena in their pure form, i.e. without the interference of factors deemed irrelevant to the matters under discussion. According to Nowakowa and Nowak (2000), idealization is a sign of the maturity a given theory has reached, and is typical of exact and natural sciences. An interesting example of idealization is Galileo's law of free fall, formulated in 1602 and presented at length in Drake's translation, published in 1962. Basically, the law states that a falling object will descend with unvarying acceleration, provided that the resistance of the medium through which it is falling is insignificant (Galileo, 1962, p. 155). In other words, the law of free fall deems a number 
of elements to be negligible, including forces acting upon a falling body, which occur in the real world but need to be discarded for the sake of the model.

In linguistics, Galileo's approach to idealization resonates in Chomsky's theory of generative grammar $(1965,1980)$. One of the most prominent examples of ideal entities inhabiting Chomskyian linguistics is the idealized native speaker, who is a member of a homogenous community consisting of other idealized exemplars. The ideal speaker-listener is a competent user of a language, resistant to possible interferences from irrelevant elements, including memory or concentration deficits (Chomsky, 1965, p. 3). Another key abstraction in Chomsky's linguistic theory is language itself, defined as an idealized system, i.e. one that is not affected by, or contaminated with, influences other than those deriving from the language faculty (Botha, 1987, p.70). The language faculty, in turn, is a computational mechanism, responsible for generating grammatically perfect forms. All that is ungrammatical, i.e. derived by capacities other than the language faculty, which includes perception, the conceptual system and the environment, is classified as an impurity and placed outside the language system (Chomsky, 1980, p. 28).

The above definition, or mode of idealization, whereby the faculty of language in its narrow sense (viewed as an independent computational mechanism) is kept distinct from the faculty of language in the broad sense (including elements of the sensory-motor and conceptual systems, and maintained separate from other organism-internal systems, including memory or respiration (see Hauser, Chomsky, \& Fitch, 2002)) is rejected by Cognitive Linguistics (henceforth also CL). ${ }^{1}$ Instead, cognitive linguists claim that the distinction between language and other general cognitive capacities needs to be blurred since the former is taken to reveal the latter (see Evans \& Green, 2006). Furthermore, cognition is seen not as located in a number of cognitive mechanisms, but as emerging from them and the other systems these cognitive mechanisms interact with, i.e. the body, the sensory-motor system, the physical environment, and the social milieu (see Barsalou, in press, p. 3). Consequently, if language reflects cognition, and if cognition is grounded in a set of interacting contexts, CL will not see the language system as abstracted from its impurities or irregularities but instead as embedded in them. This being the case, and if Cognitive Linguistics is to be viewed as a mature theory, i.e. a cognitive science, it is justifiable to inquire about its mode of idealization.

\section{Cognitive Linguistics as a Theory of Meaning}

Cognitive Linguistics is an approach to the study of language which emerged in the 1980s in English-speaking countries, mainly the USA. George Lakoff and Ronald Langacker are considered to be the pioneers of CL research, and the main tenets of the school are explicated in their monumental monographs: Women, fire and dangerous things and Foundations of cognitive grammar, respectively, both published in 1987. Nowadays, CL is referred to as, for instance, non-autonomous, post-structural, post-modern, continual, functional or anthropological, all of which highlight the open, context-oriented nature of language. Consequently, the language system, as well as its subsystems, is viewed as non-autonomous and precise distinctions between, for instance, syntax and morphology or morphology and lexis are not held.

For cognitive linguists, language is thoroughly symbolic and its basic component is a symbolic unit - a form-meaning pairing. Symbolic units are thus bi-polar, with a phonological unit constituting the form and a semantic unit defining the other pole. This tenet, known as the symbolic thesis (Langacker, 1987), entails that form cannot be studied independently of meaning, which in turn indicates that semantics is of paramount importance in CL. As Geeraerts (2006, p. 3) puts it, "[t]he foundational point [of CL] is that language is all about meaning", about linguistic meaning which is conceptual in nature. If meaning is equated with conceptualization, and if, as stated

\footnotetext{
${ }^{1}$ In order to clarify terminological distinctions adopted here, it needs to be said that Cognitive Linguistics the approach represented in this article - is one form of cognitive linguistics and should therefore be distinguished from, for instance, generative grammar.
} 
above, cognition is grounded it its contexts, the way in which symbolic assemblies are integrated in language processing results in a unit of meaning which is encyclopedic, perspectival, situated and usage-based.

The idea that linguistic meaning is encyclopedic entails that meaning involves knowledge of the world coupled with our other cognitive capacities. These capacities, as stated above, are crucially dependent on our biological properties and socio-cultural identities, which are not uniform and fixed but dynamic and dependent on actual experience. Hence, the encyclopedic nature of language implies that meaning is perspectival, since a symbolic unit triggers a particular construal or traces a unique path across the conceptual system. In other words, meaning is not only a focal part of the semantic pole of a symbolic unit, which Langacker (1987) calls its profile, but also its cognitive context, or base, which is necessary in order to understand the profile. This interdependence between the focal and non-focal content of a semantic pole entails that meaning is situated, i.e. it creates richer mental effects than simple denotational reference, and whenever CL refers to meaning-as-conceptualization what is in fact evoked is a concept together with its background, which together constitute a dynamic, distributed category (see Barsalou, 2008). The phenomenon of situatedness, or contextualization, is inextricably related to the usage-based nature of meaning proposed in CL. Briefly, since we produce and hear language in situated contexts of use, it is only natural that meaning representation encompasses both central and background elements.

Zlatev (2003) elaborates on the notion of situated semantics, outlined by Sinha and Kuteva in 1995, and argues that a contextualized utterance be "the smallest independently meaningful unit of language" (Zlatev, 2003, p. 454), within which mappings between component forms and their semantic poles are distributed rather than localized (see Figure 1).

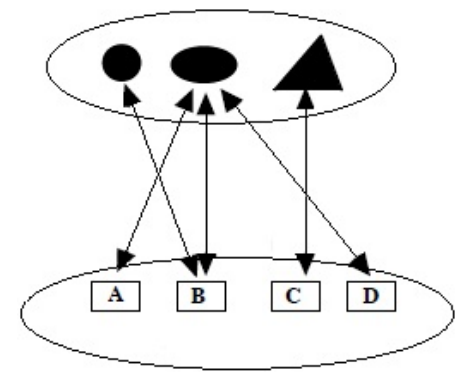

Figure 1: Situated and distributed meaning (based on Zlatev, 2003, p. 455)

As Zlatev (2003) further maintains, illustrating his claims with the semantics of prepositions, the locative meaning of the category is in fact distributed across a number of linguistic forms. Hence, mappings between semantic categories and lexemes are typically many-to-many, including conflation, distribution and composition processes. Consequently, when trying to define the meaning of form B in Figure 1, i.e. attempting to delineate a conceptualization it evokes, we need to take into account the conceptualizations related to the other forms in an utterance, which, in the case of Figure 1, are those marked A, C and D. In view of the many-to-many mappings, represented by the up-down arrows in Figure 1, an attempt to define the meaning of form $\mathrm{B}$ is in fact an attempt to characterize the meaning of its linguistic setting. Since, however, meaning in CL is taken to be usage-based, i.e., emerging from the real experience of using the language, defining the meaning of form $\mathrm{B}$ involves generalizing over its many actual settings, or contexts of use. To summarize, the semantic pole of a symbolic unit is its virtual setting, a cognitive milieu, as it were, which is idealized from the actual contexts of use. 


\section{Idealization in Cognitive Linguistics - the Problem}

The above reiteration constitutes the essence of the mode of idealization proposed in CL. What remains to be specified, however, is the type of linguistic expressions whose settings might constitute a plausible starting point for generalization. Considering the basic tenets of CL presented above, and in view of the fact that the approach explicitly rejects the method of idealization proposed by Galileo and implemented, for instance, in Chomsky's cognitive linguistics, a sound basis for constructing the architecture of idealization in CL appears to be the aforementioned language impurities, i.e. expressions which escape general rules and include, for instance, collocations or formulaic language. In this context, it is worth noting that Lakoff's early publications, i.e. Irregularity in syntax (1965/1970), Linguistic gestalts (1977), and Metaphors we live by, coauthored with Johnson in 1980, discussed irregular sentence structure, colloquial language and metaphorical linguistic expressions respectively, all of which constitute instantiations of language impurities. Thus, it seems not unfounded to postulate that in CL idealization involves constructing a model of a cognitive setting in which a particular group of symbolic units is embedded. It is therefore pseudo-idealization, i.e. generalizing over a selected, atypical set of data. According to Nowakowa and Nowak (2000, pp. 63-74), this mode of idealization was successfully applied by Darwin during his observation of species in the Galapagos Islands at the end of the 19th century, and can be formulated as follows: instead of creating ideal objects, look at a selected set of data where an analogy to idealization can be found - some factors are so insignificant that they can be neglected.

Assuming that the mode of idealization in CL is closer to Darwin's than Galileo's solution, what needs to be defined is a set of factors deemed negligible. In other words, cognitive linguists should decide, in a uniform manner, which elements of the cognitive milieu of a symbolic unit will be discarded in the process of idealization, and which ones will constitute the foundation of theorizing. However, as argued below, despite some existing methods of idealization, CL is still on its way to formulating a uniform proposal.

\section{Idealization in Cognitive Linguistics — the Solutions}

Cognitive linguists have offered a number of models which could constitute the bases of symbolic units. One of the most influential is Lakoff's Idealized Cognitive Model (ICM). As the linguist defines it, an ICM is a relatively stable representation of knowledge about a given aspect of reality, based on recurring experiences and constituting a cognitive horizon for linguistic units (Lakoff, 1987, pp. 118-135). Admittedly, an ICM is a cover-term which can be elaborated as a semantic frame or a cognitive domain (see Lakoff, 1987, p. 68).

The construct of a semantic frame was introduced by Charles Fillmore in the 1970s, and has been developed since the 1990s in the FrameNet project (n.d.). Essentially, a frame is a coherent set of concepts interrelated in such a way that in order to understand one of them, the whole background structure needs to be evoked. Thus, if one element of a frame is activated through a linguistic form related to it, all other components of the structure become automatically available as well (Fillmore, 1982, p. 111). The notion of a cognitive domain, in turn, was proposed by Langacker, who characterizes it as a general coherent knowledge structure which serves as a background against which other, more specific concepts can be defined (Langacker, 1987, p.147). A particular instantiation of a cognitive domain is a source domain, which is a relatively complex knowledge structure.

On the basis of the above descriptions of semantic frames and cognitive domains, one cannot fail to notice similarities between the two models, which is discussed at length by Croft and Cruse (2004, pp.7-32). However, there is one parallel between Fillmore's and Langacker's idealized constructs which has not been highlighted in the relevant literature. This correspondence is the fact that both a semantic frame and a cognitive (source) domain have been felicitously applied in 
the study of irregular syntactic constructions (see, for instance, Goldberg, 1995) and metaphorical linguistic expressions (see Kövecses, 2002), respectively. In other words, frames and domains have been employed to represent the conceptual content, or the semantic pole, of atypical symbolic units. Consequently, both a semantic frame and a cognitive (source) domain emerge from pseudoidealization. This being the case, what remains to be determined are, as stated above, the elements of the cognitive setting which a model will discard.

\section{Idealization in Cognitive Linguistics - the Challenge}

In order to decide whether idealization in CL has been progressing in a uniform manner, i.e. whether (ir-)relevant components of the conceptual context of a symbolic unit have been selected in a motivated and compatible way, two instantiations of pseudo-idealization will be discussed with reference to the symbolic unit anger. ${ }^{2}$ The first is a proposal based on semantic frames and the constructs of the FrameNet project, and the second is Stefanowitsch's (2006) model, founded on Kövecses' (2002) developments of Lakoff and Johnson's conceptual metaphor theory from 1980, anchored in cognitive domains.

As expected, the approaches share a number of commonalities. To begin with, both are deductive models in which the starting point for linguistic theorizing is the researchers' generalizations over selected aspects of reality. In the frame model, these are referred to as a schematic representation of a situation, while in the domain approach they constitute a naïve physics model. Secondly, both solutions highlight components of a schematic representation or a naïve model which are particularly salient. In the frame approach, these are called frame elements, whereas in the other proposal they are referred to as the main meaning focus of the domain. This stage of idealization is particularly important for the current discussion, since it involves selecting aspects of a cognitive milieu a given model sees as (in-)dispensable for the semantics of a symbolic unit.

In the case of the frame theory, 13 conceptual roles are offered to capture the semantic potential of anger, e.g. circumstances, degree, event, stimulus, topic, experiencer, expressor, or reason (https://framenet2.icsi.berkeley.edu/fnReports/data/frameIndex.xml?frame= Emotion_directed). In the domain approach, the main meaning focus of anger encompasses only 4 concepts: control, intensity, existence and harm (Kövecses, 2002). Clearly, the two theories idealize the cognitive milieu of the symbolic unit anger in dissimilar ways. These discrepancies become undisputable once the conceptual categories proposed as crucial in the cognitive setting of anger by each model are mapped onto examples of genuine language use, which, in the case of both solutions, are derived from the British National Corpus (the BNC).

In examples 1-15 below, fragments of sentence use, all taken from the BNC, are annotated using the conceptual categories proposed as non-negligible in each of the two models outlined above. Conceptual coincidences between categories in the context of the lexical unit anger proposed by the frame theory and those postulated by the cognitive domain approach are marked in red; blue signals the elements of the cognitive setting of anger proposed by the semantic frame methodology, while orange marks those put forward by the cognitive domain approach.

1. His mounting ANGER was making his speech more and more incomprehensible through his stroke-stiffened mouth. (experiencer, degree/ intensity)

2. This time, with deepening ANGER on the streets over price liberalisation, the hardliners were not to forgo their chance. (degree/ intensity, topic)

3. They have also aroused Protestant ANGER against Dr Runcie, at the same time as he has become involved in a row over his attack on the "Pharisees" of British society. (existence,

\footnotetext{
${ }^{2}$ The choice of the symbolic units anger for illustrative purposes is motivated by two factors. Firstly, its semantic analysis has been conducted within both frame and domain frameworks and hence can be reliably compared. Secondly, according to CL (Kövecses, 1986, 2002) anger tends to occur as a part of a metaphorical linguistic expression. Consequently, the symbolic unit is atypical in the sense evoked in the present discussion, i.e., it is a linguistic unit which escapes general rules.
} 
experiencer, stimulus)

4. To his own surprise all his ANGER against Edouard had evaporated. (experiencer, stimulus, existence)

5. Fear fingered Ruth again, and she suppressed a futile burst of ANGER against Adam. (intensity, stimulus)

6. Despite a flaring ANGER at this impertinence her eyes were drawn to the strong column of his throat as he swallowed. (intensity, stimulus)

7. JOHN SMITH, understandably, is boiling with ANGER at the Tory refusal to recall Parliament. (experiencer, intensity, stimulus)

8. In the Commons, Labour MPs unleashed their ANGER at the Liberal Democrats for promising to back the Government. (control, experiencer, stimulus, reason)

9. He looked away, a bitter ANGER in his eyes. (intensity, expressor)

10. Driving back, she managed to keep her ANGER in control. (control, experiencer)

11. No, better to limit the damage, control his ANGER, make nothing of it, pretend he didn't mind. (control, experiencer)

12. As he released his ANGER, he felt his love for his ex-wife, and wept over their divorce. (control, experiencer)

13. His face darkened with ANGER. (experiencer, intensity)

14. Mr Jones exploded in ANGER. (experiencer, control)

15. But his initial ANGER at finding the latest two victims of the insane killer's bloodlust, blazed into an all-consuming hatred of the man who had done it. (experiencer, stimulus, intensity)

The following implications can be drawn from the data presented above:

- Both the semantic frame model and the cognitive domain approach implement the foundational assumptions of CL on the nature of language, which is imbued with meaning, and meaning, which is a contextualized cognitive structure;

— in generalizing over the cognitive milieus of symbolic units, both frameworks neglect some contextual elements and highlight others;

- the semantic frame model and the cognitive domain approach employ theorizing through pseudo-idealization, since they both generalize over the settings of atypical symbolic units;

- there is an insignificant degree of consistency between the two proposals, since only 2 of the 33 instantiations of conceptual categories used for coding examples 1-15 above do in fact overlap (see examples 1 and 2).

These specific observations unavoidably lead to a more general conclusion. Namely, if Cognitive Linguistics aspires to become a mature scientific theory, whose constructs and hypotheses can be operationalized and falsified, it needs to develop into a more unified semantic theory, possibly choosing one of the idealization routes outlined by the two models discussed above. The solutions are presented in Figures 2(a)-2(d) below, which constitute elaborations of Zlatev's (2003) characterization of situated and distributed meaning, where B represents the lexical unit anger and A, $\mathrm{C}$ and $\mathrm{D}$ are elements of its context (see Figure 2).

The red-black path $(\mathrm{A}-\mathrm{B})$ in Figure 2(a) depicts a theoretical framework built on the convergences between the frame model and the domain approach. The orange-black path $(\mathrm{A}-\mathrm{B})$ in Figure 2(b) is a model founded on the constructs from the domain theory alone, while the blackblue path (B-C-D) in Figure 2(c) accounts for the elements of the setting proposed in the frame methodology. Finally, the rainbow path $(\mathrm{A}-\mathrm{B}-\mathrm{C}-\mathrm{D})$ would be a way of modeling the cognitive milieu of a symbolic unit which embraces the conceptual categories from both proposals (see Fig. $2(d))$. 


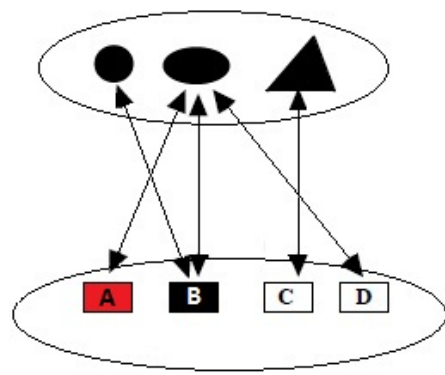

(a) The red-black path

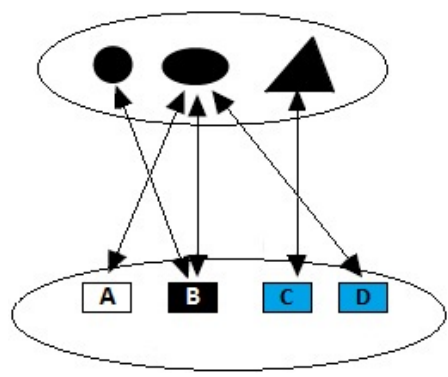

(c) The black-blue path

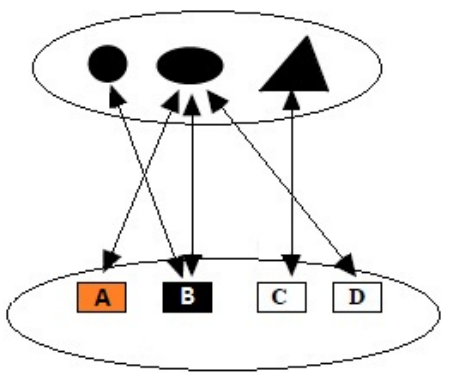

(b) The orange-black path

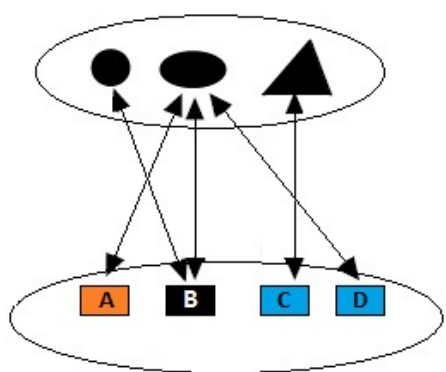

(d) The rainbow path

Figure 2: Idealization routes of Cognitive Linguistics

A guided choice of one of the above solutions, or paths of idealization, is undoubtedly a challenge which CL needs to face on its way to becoming a semantic theory in which situated and distributed nature of meaning is accounted for in a motivated manner.

\section{References}

Barsalou, L. (2008). Grounded cognition. Annual Review of Psychology, 59, 617-645. http://dx.doi. org/10.1146/annurev.psych.59.103006.093639

Barsalou, L. (to appear). Situated conceptualization: theory and application. In Y. Coello \& M. H. Fischer (eds.), Foundations of embodied cognition. East Sussex,UK: Psychology Press.

British National Corpus XML World Edition (2007). Oxford: Oxford University Computing Services.

Botha, R. (1987). The generative garden game: challenging Chomsky at conceptual combat. Stellenbosch Papers in Linguistics, 16, 1-283. http://dx.doi.org/10.5774/16-0-94

Chomsky, N.(1965). Aspects of the theory of syntax. Cambridge: MIT Press.

Chomsky, N. (1980). Rules and representations. Oxford: Basil Blackwell. http://dx.doi.org/10.1017/ S0031819100050658

Croft, W., \& Cruse, D. (2004). Cognitive linguistics. Cambridge: Cambridge University Press. http: //dx.doi.org/10.1017/CB09780511803864

Evans, V. \& Green, M. (2006). Cognitive linguistics: An introduction. Edinburgh: Edinburgh University Press.

Fillmore, C. (1985). Frames and the semantics of understanding. Quaderni di Semantica, 6(2), 222-254.

FrameNet project. (n.d.). Retrieved 20 March 2015, from https://framenet.icsi.berkeley.edu/ fndrupal/

Galileo. (1962). Discoveries and opinions of Galileo (Siderius Nuncius and Saggiatore). (S. Drake, Trans.). Berkeley: University of California Press.

Geeraerts, D. (2006). A rough guide to cognitive linguistics. In D. Geeraerts (Ed.), Cognitive linguistics: Basic readings (pp. 1-28). Berlin: Mouton de Gruyter. http://dx.doi.org/10.1515/ 9783110199901.1 
Goldberg, A. (1995). Constructions: a construction grammar approach to argument structure. Chicago: University of Chicago Press.

Hauser, M., Chomsky N., \& Fitch, W. (2002). The faculty of language: What is it, who has it, and how did it evolve? Science, 298, 1569-1579. http://dx.doi.org/10.1126/science.298.5598.1569

Kövecses, Z. (1986). Metaphors of anger, pride and love: A lexical approach to the structure of concepts. Amsterdam: John Benjamins. http://dx.doi.org/10.1075/pb.vii.8

Kövecses, Z. (2002). Metaphor: A practical introduction. Oxford: Oxford University Press.

Lakoff, G. (1970). Irregularity in syntax. New York: Holt, Rinehart \& Winston. (Reprint of Lakoff 1965)

Lakoff, G. (1977). Linguistic gestalts. Papers from the Regional Meeting of the Chicago Linguistic Society, $236-287$.

Lakoff, G. (1987). Women, fire, and dangerous things: What categories reveal about the mind. Chicago: University of Chicago Press. http://dx.doi.org/10.7208/chicago/9780226471013.001.0001

Lakoff, G. \& Johnson, M. (1980). Metaphors we live by. Chicago: University of Chicago Press.

Langacker, R. W. (1987). Foundations of cognitive grammar: theoretical prerequisites. Stanford: Stanford University Press.

Nowakowa, I. \& Nowak, L. (2000). Idealization X: The richness of idealization. Amsterdam: Rodopi. (Poznan Studies in the Philosophy of the Sciences and the Humanities, 69)

Sinha, C. \& Kuteva, T. (1995). Distributed spatial semantics. Nordic Journal of Linguistics, 18(2), 167-199. http://dx.doi.org/10.1017/S0332586500000159

Stefanowitsch, A. (2006). Words and their metaphors: A corpus-based approach. In A. Stefanowitsch \& S. Gries (Eds.), Corpus-based approaches to metaphor and metonymy (pp. 61-105). Berlin: Mouton de Gruyter.

Zlatev, J. (2003). Polysemy or generality? Mu. In H. Cuyckens, R. Dirven, \& J. Taylor (Eds.), Cognitive approaches to lexical semantics (pp. 447-494). Berlin: Mouton de Gruyter. http://dx.doi.org/10. $1515 / 9783110219074.447$

\section{Acknowledgment}

This work was supported by a core funding for statutory activities from the Polish Ministry of Science and Higher Education.

The author declares that she has no competing interests.

This is an Open Access article distributed under the terms of the Creative Commons Attribution 3.0 PL License (http://creativecommons.org/licenses/by/3.0/pl/), which permits redistribution, commercial and noncommercial, provided that the article is properly cited.

\section{(C) The Author 2016}

Publisher: Institute of Slavic Studies, Polish Academy of Sciences, University of Silesia \& The Slavic Foundation 